\title{
Les actions « logiques » et « non-logiques » selon Pareto
}

\section{Raymond Boudon}

\section{(2) OpenEdition}

12 Journals

Édition électronique

URL : http://journals.openedition.org/ress/2503

DOI : $10.4000 /$ ress.2503

ISSN : 1663-4446

Éditeur

Librairie Droz

\section{Édition imprimée}

Date de publication : 15 décembre 2013

Pagination : $19-46$

ISBN : 978-2-600-01805-0

ISSN : 0048-8046

\section{Référence électronique}

Raymond Boudon, « Les actions « logiques » et «non-logiques » selon Pareto », Revue européenne des sciences sociales [En ligne], 51-2 | 2013, mis en ligne le 01 janvier 2017, consulté le 21 décembre 2020. URL : http://journals.openedition.org/ress/2503; DOI : https://doi.org/10.4000/ress.2503 


\title{
LES ACTIONS «LOGIQUES»ET «NON-LOGIQUES» SELON PARETO*
}

RAYMOND BOUDON article posthume

Résumé. La distinction parétienne entre actions «logiques》 et «non-logiques》 reste d'une importance considérable pour les sciences sociales contemporaines: la distinction réapparaît le plus souvent sous d'autres termes, mais elle continue de jouer le rôle d'un principe organisateur. Ainsi, la théorie du choix rationnel, cœur de la théorie économique a étendu son influence sur la sociologie. À l'opposé, la théorie sociologique traditionnelle, qui insiste sur l'action de déterminismes sociaux sur le comportement, séduit les économistes dits «alternatifs». Peut-on espérer dépasser ces clivages?

Mots-clés: Vilfredo Pareto, théorie du choix rationnel, croyances, rationnalité située.

\begin{abstract}
Pareto's distinction between "logical" and "non-logical" actions reappears most often under other words, but remains of utmost importance for contemporary social sciences. The distinction takes the role of an organizing principle. Thus rational choice theory, the hart of economic theory, has extended its influence on sociology. As to the traditional sociological theory that insists on explaining human behavior by social determinism, it seduces the so-called "alternative economists". Is it possible to outweigh these cleavages?
\end{abstract}

Keywords: Vilfredo Pareto, rational choice theory, beliefs, situated rationality.

* Version révisée par l'auteur, en janvier 2013, pour la Revue européenne des sciences sociales, d'un texte initialement paru en 2000 sous le titre «Vilfredo Pareto: rationalité ou irrationalité des croyances?», (Études sur les sociologues classiques, t. II, PUF, p. 165-200) et publié en l'état conformément à ses voeux. 
Dans un article précédent, j’avais insisté sur la tension que j’avais cru percevoir entre deux aspects de la pensée de Vilfredo Pareto (Boudon, 1984). Il nous dit d'une part que les raisons que se donnent les sujets sociaux de faire ce qu'ils font ou de croire ce qu'ils croient sont - sauf s'agissant des raisons relevant de la rationalité instrumentale - un «vernis logique» travestissant les causes réelles de leurs actions et de leurs croyances, lesquelles seraient à rechercher du côté d'hypothétiques «sentiments». Étudiant avec précision les règles rhétoriques présidant aux «dérivations », il admet ipso facto d'autre part qu'on ne peut endosser n'importe quel raisonnement fallacieux, et que par suite des raisons, mêmes fausses, peuvent avoir une influence causale sur la formation de la conviction et de l'action.

Pour le sociologue contemporain, cette distinction est d'une importance particulière, car elle oppose avec une clarté remarquable deux modèles d'explication des actions et des croyances qui, de Pareto à nos jours, devaient être repris au travers d'infinies variations par les sciences sociales. Quant à la tension entre les deux modèles, elle nous concerne, elle aussi directement, car elle traverse non seulement l'œuvre de Pareto, mais les sciences sociales dans leur ensemble.

\section{L'IMPORTANCE DE LA DISTINCTION}

Le cœur de la théorie sociologique de Pareto est constitué par sa distinction entre «actions logiques » et «actions non-logiques », l'une des idées du Traité de sociologie générale (1968 [1916]) qui est le mieux passée à la postérité.

Il est important d'y revenir aujourd'hui. D’abord, elle exprime une distinction qui structure en permanence le domaine des sciences sociales dans son ensemble, au point d'en représenter une sorte de principe organisateur. C'est probablement cette congruence entre la structuration des sciences sociales et la distinction de Pareto qui explique que celle-ci ait été retenue. Ensuite, Pareto définit cette distinction avec une clarté et une netteté particulières. En troisième lieu, elle représente une sorte de carrefour conceptuel ouvrant sur des options épistémologiques et anthropologiques contrastées. Enfin, cette distinction est à la fois féconde et perverse : féconde, car elle a donné naissance à des modèles explicatifs qui n’auraient peut-être jamais vu le jour sans elle; 
perverse, parce qu'elle a contribué à balkaniser les sciences sociales, à dissiper l'espoir de les voir unifiées, à rendre la communication entre disciplines plus difficile, et aussi à installer dans les sciences sociales des postulats irréalistes. Or une discipline ne peut prétendre à être scientifique qu'à condition de viser le réalisme. En dépit de tous les constructivismes, une discipline scientifique doit chercher à décrire le réel tel qu'il est.

\section{LA DISTINCTION DE PARETO}

Selon le §150 du Traité (Pareto, I968 [1916], p. 66), «Il y a des actions qui sont des moyens appropriés au but et qui s'unissent logiquement à ce but». Pareto précise : «nous appellerons actions logiques les opérations qui sont logiquement unies à leur but, non seulement par rapport au sujet qui accomplit ces opérations mais encore pour ceux qui ont des connaissances plus étendues ».

À titre d'illustration, Pareto évoque le cas des marins grecs qui rament pour faire avancer leur trirème. Le moyen «ramer» est «logiquement» (i.e. effectivement) relié à l'objectif «faire avancer le bateau». Le moyen est objectivement bon. Il est employé parce qu'il est perçu comme tel par le rameur.

Mais le marin grec ne se contente pas de ramer. Avant de partir, il sacrifie à Poséidon, le dieu de la mer. Le marin grec perçoit ce sacrifice comme indispensable, alors qu'il est inutile. Il y a donc ici discordance entre le subjectif et le réel. Le Grec croit à l'efficacité d'un moyen qui n’en a aucune. Le sacrifice à Poséidon est une action «non-logique».

Tout est dit dans ce texte sur la distinction entre actions logiques et actions non-logiques. Soulignons cependant la nuance importante qu'introduit Pareto : «nous appellerons actions logiques les opérations qui sont logiquement unies à leur but, non seulement par rapport au sujet qui accomplit ces opérations mais encore pour ceux qui ont des connaissances plus étendues » (c'est moi qui souligne). Je reviendrai sur ce passage essentiel. 


\section{I. LA THÉORIE DE LA RATIONALITÉ DE PARETO}

Admettons que le qualificatif «logique» au sens où l'emploie Pareto, puisse être retraduit dans notre langage moderne par «rationnel». On peut dire alors que le texte que je viens de citer et dont la substance est reprise dans l'ensemble du Traité de sociologie générale esquisse une théorie de la rationalité. Celle-ci se définit par les propositions suivantes :

1) Pareto assimile rationalité et rationalité instrumentale: est rationnelle une action utilisant un moyen approprié par rapport à une fin. On peut dire encore que, pour Pareto, la rationalité est conséquentialiste. Si une action a bien pour conséquence de rapprocher l'acteur de l'objectif qu'il poursuit, cette action est rationnelle. Cela pose une première question cruciale : faut-il assimiler la rationalité à la rationalité instrumentale?

2) Dans le cas des «actions logiques» (i.e. des actions rationnelles au sens de la rationalité instrumentale), les causes des actions sont confondues avec les raisons de l'acteur. Le marin rame parce qu'il souhaite atteindre telle destination et que ramer est un moyen approprié à cette fin.

3) Dans le cas des actions «non-logiques » (i.e. irrationnelles du point de vue de la rationalité instrumentale), la question des causes des actions est plus complexe. Le marin grec croit qu'il faut faire des sacrifices à Poséidon. La cause de son action est ici une croyance fausse. Par suite, il faut remonter à la cause de cette croyance. Selon Pareto, cette cause ne peut être recherchée du côté d'un lien «logique» entre moyen et objectif puisque celui-ci n'existe pas. Elle doit donc l'être, avance-t-il, dans les mécanismes qui font croire au marin à l'existence d'une liaison objective là où il n'y en a pas. Pareto propose de voir les causes des croyances sous-jacentes aux actions non-logiques dans ce qu'il appelle des «sentiments». Il faut écrire le mot entre guillemets, car il s’agit de sentiments inobservables. 
Ces inobservables permettent de théoriser les données de l'observation, un peu comme la notion de «force» en physique. Personne n’a jamais observé les «forces» qu'évoquent les physiciens, mais cette notion permet d'organiser les données de l'expérience. Pareto traite, de même, les «sentiments » qu’il évoque pour expliquer les actions «non-logiques» comme une entité dont il lui paraît indispensable de postuler l'existence à partir du moment où l'on constate que le sujet social croit à des relations causales dépourvues de fondement objectif. Il est explicite sur ce point : ses «sentiments » sont bien inobservables, à l'instar des «forces» de la physique. Ils n'ont pas de rapport avec les sentiments ordinaires. Pour marquer la différence entre les sentiments observables (la colère par exemple) et les sentiments inobservables dont il postule l'existence, il les qualifie de «résidus», tout en prenant soin de préciser qu'il aurait pu leur donner n'importe quel autre nom.

Il faut remarquer toutefois que, à la différence des «forces» de la physique, les «résidus» de Pareto ne se sont pas imposés.

Mais il faut surtout noter que la théorie de la rationalité de Pareto effectue à ce point un saut logique, dont il ne paraît pas se rendre compte : le fait que le marin grec croit faussement que les sacrifices à Poséidon sont indispensables n'implique pas, contrairement à ce qu'il affirme, que les causes de son action ne résident pas dans des raisons. Pareto relève bien que les acteurs sociaux veulent généralement que leurs croyances, même celles qui nous paraissent les plus étranges, soient fondées, mais il ne s'arrête guère à l'idée que les raisons fausses que l’acteur se donne puissent représenter la cause réelle de ses croyances.

Les actions non-logiques se répartissent en genres, nous dit Pareto. Dans les actions non-logiques du premier genre, le sujet ne se donne pas de raisons; dans les actions du second genre, il s'en donne. Or, déclare-t-il, les actions nonlogiques du second genre sont de loin les plus fréquentes. En d’autres termes, le sujet se donne le plus souvent des raisons. Mais ces raisons sont un simple «vernis logique » lorsqu'elles sont évoquées par l'acteur se livrant à une action «non logique », comme de sacrifier à Poséidon. 
Bref, pour expliquer les actions non-logiques, Pareto postule l'existence chez l'acteur social, non seulement de sentiments conjecturaux en lesquels il propose de voir les causes réelles de ces actions, mais aussi d'un besoin de «rationalisation» au sens freudien ou de «justification» au sens dubitatif du terme. Mais il n'explique à aucun moment pourquoi il introduit le postulat selon lequel, lorsqu'une croyance repose sur des raisons fausses, celles-ci ne peuvent être les causes de ladite croyance.

Que ce postulat ne soit pas obligatoire, l'histoire des sciences le démontre. Comme Pareto le dit lui-même dans une boutade, l'histoire des sciences est celle des idées fausses auxquelles l'humanité a cru sur la foi des savants. Il est vrai qu'elle a donné naissance à une multitude d'idées fausses auxquelles on a cru; mais le savant d'hier y a cru, non parce qu'il était asservi à des «sentiments » (des «résidus ») conjecturaux, mais parce que, étant donné l'état de la science, il avait des raisons d'y croire. Lavoisier a mis la théorie du phlogistique de Priestley au rencart, mais Priestley avait des raisons de croire à sa théorie.

Pareto introduit donc ici, au cœur même de sa théorie sociologique, un postulat qui ne s'impose pas. Pourtant, il paraît le tenir pour évident. D’autre part, il ne nie pas que les causes des erreurs scientifiques résident dans des raisons fausses. Mais il ne discute pas de l'articulation qu'il serait indispensable d'établir entre sa théorie des croyances «non-logiques » et l'explication de l'erreur scientifique telle que tout historien des sciences la conçoit.

Ce point est d'importance capitale : l'objection s'adresse en effet, non seulement à Pareto, mais à toute théorie irrationnelle des croyances.

4) La théorie de la rationalité de Pareto définit - par différence- les contours de l'espace du «non-logique »; en d'autres termes : de l'irrationnel.

Étant donné que la rationalité coïncide selon Pareto avec la rationalité instrumentale, elle ne saurait concerner que les moyens. Le choix des fins se retrouve donc ipso facto rejeté dans le domaine du «non-logique». Les objectifs poursuivis par l'individu, les valeurs qui le guident ne sauraient en d’autres termes être endossés pour des raisons «logiques». 
Cette proposition débouche nécessairement, soit sur une théorie de caractère «décisionniste» (l’adhésion aux valeurs, la détermination des objectifs sont le fait de décisions «gratuites »), soit sur une théorie «causaliste» (l'adhésion aux valeurs, la détermination des objectifs sont l'effet de causes psychiques, biologiques ou sociologiques posées à titre conjectural). C'est pour la seconde solution qu'opte Pareto, lorsqu'il propose de faire de sentiments inobservables (les « résidus ») la cause des actions «non-logiques ».

5) Ce choix le conduit à une autre proposition essentielle, à savoir que la sociologie serait la discipline chargée d'élucider les causes des actions nonlogiques, tandis que l'économie aurait vocation à élucider les raisons des actions logiques. En d'autres termes, l'économie aurait pour domaine les comportements relevant de la rationalité instrumentale, la sociologie les comportements qui n'en relèvent pas.

Pour comprendre la théorie de Pareto, il est indispensable de voir que la tradition de pensée dans laquelle il s'inscrit est le positivisme. Il oppose la démarche «logico-expérimentale» à celle du métaphysicien. Il admet en d'autres termes l’idée, lancée par Auguste Comte, d’une différence de nature entre le raisonnement de caractère scientifique et le raisonnement métaphysique.

Il faut cependant préciser que, selon Pareto, le raisonnement «logicoexpérimental » n'est pas le seul fait du scientifique. L'homme de la rue, l'artiste ou le juriste agit aussi dans la vie courante de façon «logico-expérimentale», déclare-t-il. Il veut dire par là que la pensée ordinaire, comme l’argumentation juridique ou les choix de l'artiste, peuvent relever de la rationalité instrumentale. L'on peut faire la même remarque s'agissant de certains raisonnements philosophiques, et Pareto le reconnaît. Mais d'un autre côté, il retient intégralement les distinctions du positivisme entre les modes de pensée positif, d'une part, théologique et métaphysique, de l’autre. Comme Auguste Comte, il crée par différence une classe de raisonnements échappant à la rationalité instrumentale et qui, par là, ne peuvent prétendre quà une apparence de validité. 


\section{L'ACTUALITÉ DE LA DISTINCTION ENTRE ACTIONS LOGIQUES ET ACTIONS NON-LOGIQUES}

La distinction de Pareto n'est pas seulement un surgeon de la tradition positiviste. Elle est importante pour nous, car elle constitue un principe organisateur de l'ensemble des sciences sociales, voire des sciences humaines, aujourd'hui encore.

Les économistes traitent couramment les comportements comme «logiques» au sens de Pareto, tandis que les sociologues, les anthropologues ou les psychologues tendent à les interpréter comme produits, non par des intentions ou des raisons, mais par des causes biologiques, sociologiques ou psychiques s'imposant à l'individu. À l'instar de Pareto, psychologues, sociologues et anthropologues considèrent le «causalisme »' comme un ingrédient indispensable, voire caractéristique de toute science.

Ainsi, le sociologue et l'anthropologue se contentent couramment d'évoquer les effets de socialisation pour expliquer que le marin grec sacrifie à Poséidon avant de prendre la mer. Et lorsque le psychologue cognitif crée des situations de problem solving qui engendrent des réponses erronées de la part des répondants, il interprète facilement ces erreurs comme dues à la présence de «biais cognitifs», c'est-à-dire de mécanismes logiques défaillants.

Ainsi, Richard Shweder (1977) interprète les erreurs d'induction statistique couramment commises par la pensée spontanée comme dues au fait que cette dernière suivrait des règles dépourvues de fondement, mais qui s’imposeraient au sujet à son insu, et auxquelles il donne le nom de «pensée magique ». Selon cette manière de voir, la pensée ordinaire serait donc le produit d'une organisation cérébrale qu'on ne peut observer directement, mais dont on peut inférer l'existence à partir de ses effets. Les schémas d’analyse proposés par Pareto (et par d'autres auteurs classiques, comme Lucien Lévy-Bruhl) continuent donc bien d'inspirer nombre d'analyses contemporaines.

I Entendant par là la doctrine diffuse selon laquelle les causes que l'homme de science peut légitimement invoquer ne sauraient être des raisons et devraient être de préférence «matérielles » et cachées, i.e. saisissables seulement de façon indirecte. 
En un mot, les explications du comportement proposées par la psychologie, la psychologie cognitive, l’anthropologie ou la sociologie sont très généralement causalistes, tandis que celles de l'économie sont généralement rationnelles au sens instrumental.

La distinction proposée par Pareto décrit donc bel et bien une sorte de postulat fondamental présidant à l'organisation des sciences sociales en deux groupes distincts.

\section{I. FRAGILITÉ DE LA DISTINCTION}

En même temps, cette distinction est discutable. On le voit à ce que les explications rationnelles des économistes sont facilement jugées superficielles par les psychologues, les sociologues et les anthropologues. Mais les explications irrationnelles du comportement qui ont la faveur de l'autre camp s'exposent de leur côté à l'objection que les mécanismes dont elles postulent l'existence constituent des boîtes noires impossibles à ouvrir. Quand on a dit que les marins grecs sacrifiaient à Poséidon sous l'effet de «sentiments» inobservables, a-t-on expliqué quoi que ce soit? Quand on déclare que les marins grecs croyaient à Poséidon par un effet de «socialisation» parce qu’ils avaient été élevés dans cette idée, a-t-on expliqué quoi que ce soit? La notion de socialisation n'est pas vide de sens, mais suffit-il de l'évoquer pour expliquer que les marins grecs croyaient à Poséidon? Suffit-il de mettre les dérapages de l'inférence statistique dont témoigne la pensée ordinaire sur le compte de «biais cognitifs» pour les expliquer?

Bref, à travers sa distinction entre actions logiques et actions non-logiques, Pareto soulève la question fondamentale, toujours débattue dans les sciences sociales d'aujourd'hui, des représentations qu'elles sont en droit de se donner du sujet social. La pertinence de la distinction est reconnue par tous. En même temps, elle débouche sur des difficultés considérables. Les sociologues veulent que les actions que les économistes considèrent comme «logiques» soient «nonlogiques »; les économistes que les actions que les sociologues considèrent comme «non-logiques» soient en réalité «logiques». Tous admettent la pertinence de la distinction. Mais un conflit chronique oppose les deux camps sur les comportements qu'il faut placer dans les deux cases. Les «nouveaux économistes» veulent 
que le crime et le suicide lui-même soient des actions «logiques». Les sociologues traditionalistes veulent que les comportements d'échange et de consommation les plus banals traduisent des effets de socialisation.

Je ne veux pas entrer dans une discussion des paradigmes que je viens d'évoquer. Plus simplement, je voudrais développer quelques-unes des critiques majeures que l'on est en droit de formuler à l'encontre de la distinction de Pareto.

\section{LA RELATION ENTRE MOYENS ET FINS}

À partir du moment où la validité d'une relation entre moyen et fin ne peut être appréciée que si l'on dispose de certaines connaissances, on ne peut déclarer une action logique ou non-logique dans l'absolu, mais seulement relativement aux connaissances dont dispose l'acteur.

En effet, si une proposition de type «M est un moyen adéquat pour atteindre l'objectif $\mathrm{O} »$ peut dans certains cas être facilement établie, elle peut aussi supposer une accumulation préalable de connaissances. Ces connaissances peuvent être disponibles en $\mathrm{t}+\mathrm{k}$ mais non en $\mathrm{t}$, de sorte que le jugement « $\mathrm{M}$ est un moyen adéquat pour atteindre l'objectif $\mathrm{O}$ » pourra être perçu comme vrai en $t$ et comme faux en $t+k$ et réciproquement. Les connaissances en question peuvent être disponibles dans tel contexte social C, mais non dans le contexte C'. En conséquence, le jugement «M est un moyen adéquat pour atteindre l'objectif $\mathrm{O}$ » peut passer pour vrai en $\mathrm{C}$ et faux en $\mathrm{C}$ ' et réciproquement. Pareto est conscient de cette difficulté. C’est pourquoi il évoque le jugement de «ceux qui ont des connaissances plus étendues». Il sait bien que la seule manière de juger de la validité objective du jugement «M est un moyen adéquat pour atteindre l'objectif $\mathrm{O}$ » consiste à consulter ceux-là «qui ont des connaissances plus étendues». Mais ces derniers risquent d'être remplacés demain par des experts ayant des connaissances «plus étendues» encore.

Pareto ne paraît pas prendre en compte ces remarques élémentaires. Or elles comportent des conséquences d'une portée considérable. 
La principale est qu'un moyen peut être jugé irrationnel par l'observateur, mais ne pas l'être pour l'acteur lui-même, dès lors que celui-ci ne dispose pas des «connaissances plus étendues» de l'observateur.

Contrairement à la théorie de Pareto, mais en accord avec son évocation de la notion de compétence, il est donc difficile de qualifier une action d'«irrationnelle » à partir du moment où, pour l'acteur, son action lui paraît fondée sur des raisons solides eu égard aux «connaissances» dont il dispose.

\section{I. LA RATIONALITÉ SITUÉE}

Ce point essentiel a par contre été parfaitement vu par Max Weber dans ses textes relatifs à l'explication des croyances magiques.

Les actes motivés par la religion ou la magie sont des actes, au moins relativement, rationnels, en particulier sous leur forme primitive. [...] Les étincelles produites en frottant le morceau de bois sont des effets tout aussi 'magiques' que la pluie produite par le faiseur de pluie (Weber, I97 I [1922], p. 429-430).

Malgré son caractère lapidaire, le sens de ce texte n'est pas difficile à restituer. Pour nous, observateurs, le comportement du faiseur de feu n'est pas magique, à la différence de celui du faiseur de pluie. Mais nous faisons cette différence parce que nous savons que l'énergie cinétique peut se transformer en énergie thermique. Nous comprenons donc facilement qu'une manière d'obtenir la combustion d'un morceau de bois consiste à le frotter contre un autre morceau de bois. Nous percevons l'action du faiseur de feu primitif comme «logique» au sens de Pareto, parce qu'elle nous apparaît comme un moyen objectivement bien adapté d’atteindre l'objectif recherché : faire du feu. Et nous percevons celle du faiseur de pluie comme «non-logique», parce qu'elle ne correspond pas à une relation de causalité objectivement valide.

Si l'on se place maintenant du point de vue de l'acteur primitif, nul ne lui a enseigné les lois de la transformation de l'énergie. Et il est peu vraisemblable que son intuition lui permette de pressentir une théorie que la physique occidentale a mis des siècles à établir. 
Comme Weber l'affirme, l'action du faiseur de feu n'est pas perçue par cet acteur comme d'une nature différente de celle du faiseur de pluie. Dans les deux cas, l'action est fondée dans son esprit sur des raisons qui lui paraissent solides et qui ne le sont pas pour nous. «Le comportement du faiseur de feu est tout aussi magique que celui du faiseur de pluie» en ce sens que le comportement du premier est fondé dans l'esprit de l'acteur sur des considérations certainement aussi peu satisfaisantes du point de vue de l'observateur que le comportement du second.

Une application littérale de la théorie de Pareto aboutirait en revanche ici à des difficultés considérables. Elle conduirait à classer comme «logique» l’action du faiseur de feu et comme «non-logique» celle du faiseur de pluie, à faire relever la première de l'économie et la seconde de la sociologie, à voir dans des raisons objectives la cause de la première et dans des «résidus», la cause de la seconde. Mais ce serait là, non seulement multiplier les hypothèses fragiles, mais donner dans le sociocentrisme. La distinction faite par l'observateur entre le «faiseur de pluie» et le «faiseur de feu» est en effet une conséquence de l'état de son propre savoir.

Pour mesurer le caractère difficilement acceptable de la distinction de Pareto, il suffit d'évoquer les théories que nous jugeons fausses aujourd'hui et qui furent jugées vraies hier. Personne ne songerait à faire du lamarckisme une théorie «non-logique». Il était fondé sur des raisons perçues comme solides par ceux qui les endossèrent, car ils ne pouvaient naturellement mesurer leur faiblesse à l'aune de connaissances qui ne devaient apparaître qu'ultérieurement, avec Charles Darwin. On peut faire les mêmes remarques s'agissant des actions fondées sur des théories périmées. Alexandre entraîna ses armées jusqu'en Inde parce qu'il pensait y atteindre les limites du disque terrestre.

Ces exemples montrent qu'on peut endosser le jugement «M est un moyen adéquat pour atteindre l'objectif $\mathrm{O} \gg$ avec des raisons qui paraissent adéquates à l'acteur, mais non à l'observateur, et réciproquement. Tout dépend des connaissances de l'un et de l'autre. On doit en un mot concevoir la rationalité cognitive comme «située», cela n'impliquant aucune conclusion relativiste. Le fait de traiter les croyances du faiseur de pluie comme non irrationnelles n'implique pas que ses croyances soient aussi valides que celles de l'observateur. 


\section{LA RATIONALITÉ COGNITIVE}

On peut opposer à Pareto les intuitions de certains sociologues et anthropologues: on pense ici à Émile Durkheim dans certains passages des Formes élémentaires de la vie religieuse (I9I2), à Edward E.Evans-Pritchard (1972 [1937]), à Robin Horton (1982) ou à Michel Spiro (1995 [1987]). Tous proposent plus ou moins explicitement une théorie cognitiviste des croyances : ils partent en d'autres termes de l'hypothèse que les croyances, vraies ou fausses, sont l'effet de raisons perçues comme solides par les sujets sociaux et faisant par suite sens pour eux.

Si cette théorie rencontre de grandes résistances, c'est en raison surtout de l'influence de la tradition causaliste que j'évoquais il y a un instant, et aussi parce que nous sommes à ce point imprégnés de culture scientifique que nous avons du mal à concevoir que d'autres cultures aient pu croire effectivement à des théories que nous percevons comme absurdes, comme les rituels des marins grecs. Ce sociocentrisme explique qu'on perçoive certaines croyances comme étant «non-logiques ».

Il permet aussi de comprendre que, s'agissant de l'explication des croyances dépourvues de fondement objectif, on observe de façon récurrente trois explications typiques, alors que deux d'entre elles ne résistent guère à la critique.

\section{I.TROIS TYPES D'EXPLICATION DES CROYANCES «NON-LOGIQUES»}

1) La première est celle qu'illustrent par exemple Ludwig Wittgenstein ou un historien contemporain de l'Antiquité classique, Paul Veyne.

Elle part de l'hypothèse que les acteurs sociaux qui paraissent endosser des croyances «non-logiques » ne croient pas en réalité à ce qu’ils affirment croire, que leurs croyances ne doivent pas être interprétées comme réalistes, mais comme symboliques. Wittgenstein (1975) affirme que les rituels «magiques» ne traduisent pas des croyances en des relations de causalité illusoires, mais sont l'expression symbolique de désirs. Les rituels mimant la pluie ne viseraient pas à l'efficacité, mais traduiraient le désir de ceux qui les pratiquent de voir tomber la pluie. Veyne (1983) développe des idées proches de celles de 
Wittgenstein lorsqu'il pose la question : Les Grecs ont-ils cru à leurs mythes? Sans aller aussi loin que Wittgenstein, il se contente de suggérer prudemment que les Grecs n'ont peut-être pas cru à leurs mythes.

En fait, il faut voir surtout dans ce genre de théories un moyen permettant de dissiper le malaise que nous inspirent des croyances qui nous paraissent absurdes. Déclarer ou suggérer que les acteurs ne croient pas en réalité à ce qu'ils paraissent croire, c'est effectivement une manière de résoudre l'énigme posée par ces croyances en l'évacuant.

À ce type de théorie on peut opposer les remarques simples mais convaincantes de Hans Albert (1997) et de Robin Horton (1982): on ne prie pas un Dieu dont on ne croit pas qu'il existe, déclare justement Albert; d'où il suit qu’on ne peut accepter facilement de sacrifier à Poséidon si on ne croit pas à son existence. Les Africains affirment sans ambiguïté, dès qu’on leur pose la question, que les rituels magiques sont indispensables à l’agriculture, observe de son côté Horton.

Les théories de type «Wittgenstein» ont le mérite d'être réfutables. Mais elles ont aussi le défaut d'être réfutées : elles sont incompatibles avec les observations que nous livrent les ethnologues.

2) Le deuxième type de théories, sans doute le plus répandu et le plus influent, a, lui, par rapport au premier, l'«avantage » - si l'on peut dire - de ne pas être réfutable. Il est illustré par Pareto, mais aussi par de très nombreux auteurs, comme Lévy-Bruhl hier et, aujourd'hui, Clifford Geertz (1984) et bien d’autres anthropologues et sociologues. Ces théories supposent que les croyances sont le produit de processus psycho-sociologiques conjecturaux qui amènent le sujet à raisonner à partir de règles d'inférence objectivement non valides.

3) La troisième explication, celle d'Evans-Pritchard, de Weber, de Durkheim, ou aujourd'hui d'Horton ou de Spiro, consiste à admettre que les croyances qui nous paraissent irrationnelles sont le produit d'un effort rationnel pour interpréter le monde avec les ressources cognitives dont on dispose. C'est aussi la mienne, si l'on mautorise cette indication. 


\subsection{L'EXPLICATION COGNITIVISTE DES CROYANCES «NON-LOGIQUES»}

Dans une société «primitive» ou «sans écriture» (comme on dit plus volontiers aujourd'hui), vivant par exemple de l'agriculture, on ne peut éluder le problème des moyens permettant de faciliter le processus de croissance des plantes. Une telle société a accumulé au cours du temps toutes sortes de savoirfaire empiriques. Mais elle ne saurait tirer de l'expérience une théorie de la naissance, de la croissance, de la dégénérescence ou de la reproduction des végétaux. Or une telle théorie est indispensable pour mettre en ordre les données de l'expérience. Elle sera normalement, ainsi que le suggère Durkheim, tirée du savoir tenu pour légitime dans la société en question, en l'occurrence des doctrines religieuses en vigueur. Bref, le «primitif» tirera normalement de ses croyances religieuses toute une «biologie végétale».

Durkheim a proposé une explication convaincante du fait que les recettes magiques tirées des doctrines religieuses ne sont pas repoussées par le sujet social en dépit des échecs répétés auxquels elles conduisent : à savoir quavant de rejeter une théorie qui semble infirmée par l'expérience, on cherche normalement à neutraliser cet échec en recourant à des hypothèses auxiliaires. Cette démarche est courante dans la vie scientifique. Et elle est courante parce qu'elle est fondée: comme l'indique la thèse dite de «Duhem-Quine», lorsqu'une théorie est contredite par les faits, on ne sait en effet jamais d'avance si cette contradiction ne peut être éliminée par une modification mineure de la théorie. Il est donc sage, nous dit cette thèse, de continuer de croire à une théorie qui échoue, et d'essayer de la ravauder à l'aide d'hypothèses auxiliaires, du moins jusqu'à ce que, les objections et difficultés s'accumulant, il faille accepter qu'elle soit remplacée par une théorie meilleure. Encore faudra-t-il que ladite théorie soit imaginée. Pourquoi, demande Durkheim, anticipant par cette question la thèse de Duhem-Quine, le «primitif » devrait-il être plus rigoriste que le savant?

Finalement, Durkheim - et d'autres - nous suggèrent qu'on peut se dispenser d'introduire une discontinuité radicale entre la prière à Poséidon et l'acte de ramer. Les Grecs pensaient probablement que les deux étaient indispensables et ils ont selon toute vraisemblance effectivement cru à leurs mythes. 
Evans-Pritchard ou Horton indiquent clairement que les individus qu'ils ont observés leur apparurent toujours convaincus que leurs actions magiques ou rituelles étaient indispensables, même s'ils reconnaissaient qu'elles ne pouvaient suffire à garantir les effets recherchés.

Il n'y a donc pas de raisons d'expliquer les croyances en l'efficacité des rituels et des pratiques magiques de manière fondamentalement différente de celle qui est utilisée pour expliquer la croyance des physiciens du Moyen Âge à l'existence des «tourbillons». Ils étaient convaincus qu'il n'est pas de mouvement physique sans force qui le produise : une idée qui n’a évidemment rien d’absurde. Partant de cette idée, ils en tiraient la conclusion que le mouvement de la flèche qui a quitté l'arc ou du bateau qui continue de glisser sur son erre une fois que les voiles ont été amenées est imputable à une force, et ils cherchèrent de façon compréhensible à identifier la force en question. C'est ainsi qu’est née la théorie des «tourbillons». Elle est fausse et nous la percevons spontanément aujourd'hui comme absurde. Pourtant, elle ne l'est ni plus ni moins que les théories magiques. Les tourbillons n'existent pas davantage que Poséidon. Or, pour expliquer qu'on ait pu y croire, personne ne songerait à évoquer de mystérieuses causes affectives, comme les «résidus » de Pareto, ou les montages neurologiques qu'impliquent les «biais » évoqués par les psychologues cognitifs et par les sociologues fidèles à l’inspiration de Lévy-Bruhl. Il suffit d'admettre que les physiciens du Moyen Âge ont cherché avec les moyens dont ils disposaient à concilier une proposition qui leur paraissait s'imposer et qui s'impose toujours à nous, à savoir qu'il n'est pas de mouvement sans force qui le produise, avec certaines observations également irrécusables, comme le fait que le bateau continue d'avancer alors que le vent ne le pousse plus.

Je précise que, si les «sentiments» de Pareto ne constituent sans doute pas la meilleure explication des croyances magiques, je n'affirme ici en aucune façon que les affects ne jouent aucun rôle dans l'explication des croyances. Il est clair que les croyances magiques reposent au contraire sur des besoins existentiels normalement perçus par le sujet sur le mode affectif. C'est parce que la pluie est vitale dans une société agraire que le magicien pratique des rituels destinés à la faire tomber. Mais si les affects peuvent expliquer l'existence des 
intérêts cognitifs des acteurs sociaux, ils ne peuvent déterminer le contenu des réponses suscitées par ces intérêts cognitifs. La position que je défends ici n’est donc en aucune façon de caractère «intellectualiste »: elle ne méconnaît pas l'importance des affects, mais récuse le fait qu'ils puissent rendre compte à eux seuls du contenu des croyances collectives.

Il ne suffit pas non plus pour expliquer une croyance collective de supposer qu'elle est transmise sous l'effet de la socialisation. Une telle hypothèse est à la fois creuse et fausse : creuse, car verbeuse ; fausse, car aucun procédé de conditionnement n’aurait pu - par exemple - faire revivre l'ancienne croyance aux « tourbillons » à partir du moment où les énigmes qu'ils étaient censés dissiper ont été résolues par la découverte du principe d'inertie, c'est-à-dire où l'on eut compris que, s'il n'est pas de mise en mouvement sans cause, il n'est pas non plus d'arrêt d'un mouvement sans une cause qui produise cet arrêt. Il résulte du principe d'inertie que le mouvement du bateau qui continue de glisser sur son erre lorsque le vent est tombé n'est dû à aucune cause. Cette proposition, qui nous paraît banale, resta au sens propre du mot impensable tant que le principe d'inertie n’avait pas été formulé, car elle contredisait l'«évidence» selon laquelle «il n'existe pas de mouvement sans cause».

Bref, la croyance aux tourbillons, la croyance à l'efficacité des rituels magiques ou des prières à Poséidon peuvent s'expliquer de la même manière, en évitant tout recours à des causes conjecturales. Il suffit de remarquer, à la suite de Weber ou de Durkheim, qu'on ne peut arriver du jour au lendemain à des théories biologiques satisfaisantes sur la croissance et la dégénérescence des plantes et qu'on peut en même temps avoir un besoin urgent de telles théories.

Derechef, l'inadéquation des théories irrationalistes des croyances se révèle immédiatement à ce que personne ne songerait à les utiliser pour expliquer des croyances scientifiques qui nous paraissent évidemment fausses aujourd'hui et qui passaient pour vraies hier. Pourquoi faudrait-il les appliquer à d'autres types de croyances? Malheureusement, les théories qui évoquent des causes «profondes » passent souvent pour plus scientifiques, peut-être parce qu’elles 
ressemblent alors davantage aux théories physiques classiques, celles qui évoquent des inobservables, comme la notion de «force $» .^{2}$

À côté de la rationalité instrumentale, il faut donc introduire une autre forme de rationalité, qu'on peut qualifier de «cognitive». Elle est à l'œuvre dans l'histoire des sciences. Mais on peut supposer qu’elle est plus généralement responsable de beaucoup de croyances collectives. Cette hypothèse est celle de Weber, lorsqu'il suggère que la théologie, le droit ou l'économie ne sont pas moins soumises à un processus de «rationalisation diffuse » (Durchrationalisierung) que les sciences de la nature ${ }^{3}$. Elle est également suggérée par Durkheim, lorsqu'il évoque l'hypothèse d'une continuité entre la religion et la science.

On remarquera encore que la théorie cognitiviste des croyances que je propose ici s’applique également aux croyances mises en évidence par toutes les disciplines, qu'il s'agisse de l'anthropologie, de l'histoire des sciences et des idées, ou de la psychologie cognitive. J’ai tenté de montrer ailleurs que les «erreurs » d'inférence observées par la psychologie cognitive peuvent être facilement expliquées par une théorie de ce type. ${ }^{4}$

Peut-être Pareto a-t-il entr’aperçu les difficultés de l'hypothèse selon laquelle il faudrait imputer les croyances «magiques» à des «résidus» inobservables. Lorsqu'il évoque, dans le passage que je citais plus haut, «ceux qui ont des connaissances plus étendues », il suggère en effet, redisons-le, qu’une croyance ne peut être dite «logique» ou «non-logique» en soi, mais seulement par rapport à un certain état du savoir, individuel et collectif. Mais il ne tire pas, loin de là, toutes les conséquences de cette remarque.

2 C'est une attitude de ce genre qui paraît animer Dan Sperber (1997).

3 Philippe Steiner (1998) a appliqué cette hypothèse cognitiviste à l'histoire des idées économiques. Sur l'importance des notions de «rationalisation» et de «rationalisation diffuse» (Durchrationalisierung) chez Weber, voir le livre de référence de Michael Sukale (2002), qui consacre de nombreuses pages à ces notions cardinales dans la pensée de Weber.

4 Voir Boudon, 1995, 2011, 2014. 


\section{LES CROYANCES NORMATIVES}

La théorie de Pareto comporte une autre difficulté essentielle : les croyances normatives qui ne peuvent s'expliquer à partir de la rationalité instrumentale sont, dans sa théorie, rejetées en bloc du côté du non-logique.

Cette thèse, qu'il affirme plutôt qu'il ne la démontre, résulte sans doute d'abord dans son esprit de l'adage selon lequel on ne saurait tirer l'impératif de l'indicatif. Les raisonnements «logico-expérimentaux» utilisant toujours le mode de l'indicatif, les raisonnements débouchant sur une conclusion à l’impératif sont donc nécessairement dépourvus de validité. Elle résulte aussi de ce que, si certaines propositions normatives relèvent d'une démonstration «logico-expérimentale» (comme : «mieux vaut prévenir que guérir»), cela n'est pas vrai de toute proposition normative. Mais Pareto ne s'explique guère sur les raisons qui le poussent à rejeter les théories normatives échappant au registre du «logico-expérimental» du côté des théories dépourvues de fondement et à les traiter comme n’ayant d'autre fonction que celle de dissimuler des «résidus» sous un «vernis logique »: celui des «dérivations », c'est-à-dire des raisonnements fallacieux à partir desquels le sujet croit pouvoir se convaincre du bien-fondé de ses croyances.

Pareto n’a dans le Traité que sarcasmes contre la philosophie morale dans son ensemble et contre Emmanuel Kant en particulier. Il ne procède en aucune façon à une critique de Kant dans les développements qu'il lui consacre et y voit seulement une illustration de sa propre théorie des «résidus » et des «dérivations» (Pareto, I968 [1916], §I514). Kant prétend démontrer qu’il ne faut pas mentir. En fait, nous dit Pareto le raisonnement de Kant se réduit, une fois déployé, à une simple affirmation. Cette affirmation est l'effet des «résidus » qui habitent Kant à son insu, lesquels le poussent par exemple à détester le mensonge, et non des «dérivations» qu'il produit pour la justifier. Sa démonstration, nous dit Pareto, n'est qu'une pseudo-démonstration, visant à recouvrir d'un «vernis logique » ce qui n'est qu’une simple affirmation. L’idée que la théorie de Kant puisse avoir une valeur en elle-même n’est pas envisagée par Pareto. 
S’il est vrai qu'on ne saurait tirer l'impératif de l'indicatif, on peut construire des raisonnements aboutissant à des conclusions à l'impératif, pourvu qu'il existe dans les prémisses au moins une proposition à l'impératif. Un raisonnement peut donc déboucher sur une conclusion normative et se révéler valide ou invalide. Sans doute un raisonnement de ce type est-t-il toujours conditionnel dans la mesure où il dépend de la validité des prémisses. Mais il en va ainsi de tout raisonnement, et pas seulement des raisonnements normatifs.

Un raisonnement normatif conditionnel est par exemple le suivant: à supposer qu'on préfère circuler en ville en moyenne plutôt plus vite que moins vite, les feux rouges sont une bonne chose; il vaut mieux les conserver que les éliminer. Ce raisonnement conditionnel est parfaitement solide. Il met en jeu une relation moyens-fins au-dessus de tout soupçon. Pareto le reconnait. Il sait que, lorsque le normatif se limite au registre conséquentialiste, il relève de la rationalité instrumentale. Les jugements normatifs relevant de cette catégorie tombent donc dans la catégorie du «logico-expérimental». Comme j’ai déjà eu l'occasion de le remarquer, il reconnaît que le raisonnement juridique ou le raisonnement moral peuvent relever du «logico-expérimental» : en d’autres termes, qu'il peut exister des raisonnements juridiques ou moraux valides.

Si Pareto s'en prend particulièrement à Kant, c'est finalement parce que, à la différence notamment des utilitaristes qui proposent de fonder tout jugement normatif sur un raisonnement instrumental conséquentialiste, Kant est l'un des théoriciens du normatif, le plus notoire sans doute, qui refusent cette assimilation. Or une théorie normative non conséquentialiste ne peut relever selon Pareto que du «non-logique»; elle est en effet condamnée à sortir du cadre du «logico-expérimental».

\section{I.PARETO ET L'UTILITARISME}

L'intérêt constant qu'a suscité l'utilitarisme, son retour en force aujourd'hui via la Théorie du Choix Rationnel, le fait qu'il réapparaisse de manière récurrente en dépit des objections qui lui ont été opposées provient de ce qu’il promet d'éliminer les difficultés soulevées par l'explication des croyances normatives et généralement axiologiques. Si l'on peut, comme le propose 
l'utilitarisme, les réduire toutes à la rationalité instrumentale, c'est l'axiologique dans son ensemble qui relève alors du «logico-expérimental» et les difficultés en question s'évanouissent.

La séduction exercée par l'utilitarisme d'inspiration marxiste ou nietzschéenne provient pour partie des mêmes sources que celle de l'utilitarisme d'inspiration benthamienne : celle qu'illustre la Rational Choice Theory. Réduire la morale à la volonté de puissance des dominants ou au ressentiment, c'est aussi faire rentrer la morale dans un cadre conséquentialiste. Friedrich Nietzsche explique les sentiments moraux du sujet par les effets psychiques bénéfiques qu'ils entraînent chez lui, Karl Marx par leurs effets positifs sur les intérêts de classe du sujet. Comme l'a bien vu Weber (1920-192I), de telles conjectures ne peuvent avoir qu’une validité très réduite. Elles doivent leur pouvoir de séduction à ce qu'elles se présentent abusivement comme une théorie générale du normatif.

Comme Weber, Pareto refuse la prétention de l'utilitarisme à unifier l'explication des croyances axiologiques. Les valeurs - certaines d'entre elles du moins - ne peuvent, selon lui, être expliquées de manière «logico-expérimentale », i.e. instrumentale conséquentialiste.

Mais un raisonnement normatif de caractère non «logico-expérimental», ne saurait être qu'un «vernis logique»; il faut donc que les jugements de valeur, ne pouvant provenir de raisons, soient l'effet de «résidus» inobservables, mais dont il est nécessaire de postuler l'existence. La théorie kantienne de la raison pratique ne peut donc être qu'une pseudo-démonstration se résumant en fin de compte, selon Pareto, au précepte: «fais ce qui plaît à Kant».

Il n'est pas difficile de trouver des exemples de réactions axiologiques qui apparaissent comme des défis à la fois aux utilitaristes ou, plus généralement, à ceux qui veulent expliquer les phénomènes axiologiques dans le cadre de la rationalité instrumentale, et aussi à ceux qui, comme Pareto, proposent d'y voir l'effet de causes irrationnelles. J'évoquerai deux exemples. 


\section{I.I. La corruption politique}

Considérons d'abord le cas de la corruption politique. Il est clair que le public la supporte très mal. Elle revêt une grande importance dans les décisions des électeurs. Pourtant, la corruption est pratiquement dépourvue de conséquences sur le bien-être du citoyen, tant qu'elle ne dépasse pas un certain seuil. Sans doute n'est-elle pas entièrement dépourvue d'effets du point de vue économique : elle augmente le coût de certains biens et services et alourdit le poids de la fiscalité. Mais ces effets sont infimes et en tout cas parfaitement insensibles à l'échelle de l'électeur individuel. Ce n'est donc pas en raison des conséquences que la corruption entraîne ou risque d'entraîner sur lui-même que l'électeur lui accorde de l'importance. Les hypothèses de caractère égoïste généralement introduites pas les utilitaristes («l'individu se soucie au premier chef de ce qui lui arrive à lui-même ») apparaissent donc comme immédiatement disqualifiées, s'agissant d'expliquer la sensibilité de l'électeur à la corruption.

Faut-il alors attribuer cette aversion au fait que l'électeur serait sensible aux conséquences collectives de la corruption? On peut en effet étendre les hypothèses utilitaristes et admettre que l'individu se soucie, non seulement des états de choses qui l'affectent directement, mais aussi de ceux qui affectent son milieu social. Or, lorsque la corruption se maintient à un niveau «acceptable», ces conséquences collectives ne sont guère sensibles, elles non plus.

On peut même aller plus loin dans le raisonnement et reconnaître que la corruption peut comporter des conséquences heureuses. En France, sans la corruption, un certain nombre de municipalités qui brillent par les manifestations culturelles ou sportives qu'elles sont capables d'organiser seraient restées obscures.

Si l'on veut expliquer la sensibilité aiguë dont le public fait preuve à l'égard de la corruption il faut donc renoncer, non seulement aux hypothèses individualistes des utilitaristes, mais plus largement aux hypothèses de caractère conséquentialiste. En effet, ce ne sont ni les conséquences individuelles ni les conséquences collectives de la corruption qui font que celle-ci est condamnée par le public. La seule théorie conséquentialiste, à première vue acceptable, serait celle qui consisterait à affirmer que le rejet de la corruption s'explique par 
la peur que celle-ci ne se développe à un point tel qu'elle entraînerait alors des conséquences individuellement et/ou collectivement négatives et sensibles. Or une telle hypothèse aurait non seulement un caractère ad hoc, mais elle déboucherait en outre sur la question difficile de savoir pourquoi ce sentiment de peur est vécu, non pas comme un sentiment de peur, mais comme un sentiment d'indignation? Comment expliquer cette «fausse conscience »?

En fait, le rejet de la corruption provient de ce que le public a en tête, plus ou moins clairement et plus ou moins consciemment, une théorie de ce que doit être une bonne société. Cette théorie veut qu'une rétribution soit l'effet d'une contribution. Ce principe entraîne qu'un acteur politique ne puisse utiliser sa position pour tirer des avantages unilatéraux. Ainsi, un maire est censé assumer un certain nombre de tâches. En contrepartie, si ses électeurs sont satisfaits, il recevra de leur part gratitude, reconnaissance, éventuellement admiration; il augmentera ses chances d'être réélu s'il se représente, etc. Mais on lui reprocherait vivement d'utiliser sa position pour en tirer des avantages personnels. En amont de ces principes, on en trouve d’autres, plus généraux, comme le fait que les institutions réglant la vie sociale doivent être perçues comme légitimes, c'est-à-dire implicitement approuvées par tous. Prises ensemble, ces propositions forment un ensemble hiérarchisé, une «théorie». Or la corruption contredit cette théorie. La corruption est en d'autres termes normalement perçue comme mauvaise parce qu'elle apparaît au sujet social incompatible avec une théorie de l'ordre social qu'il a toutes chances d’approuver parce qu'il la perçoit comme fondée sur des raisons fortes.

\section{I.2. Le paradoxe du vote}

Le cas du vote fournit un autre exemple intéressant à partir duquel les théories du normatif peuvent être mises à l'épreuve (Boudon, 1997). Il existe une littérature très abondante sur le «paradoxe du vote», car le simple fait que les gens votent oppose un redoutable défi à toutes les explications du comportement de caractère conséquentialiste. En effet, la probabilité pour que mon vote affecte le résultat du scrutin est quasiment nulle. Le fait de déposer ou non un bulletin dans l'urne est donc sans conséquence. 
De nombreux articles tentent de contourner cette donnée de base de façon plus ou moins ingénieuse, mais aucune théorie conséquentialiste et a fortiori aucune théorie utilitariste du vote n’a jamais réussi à produire une explication convaincante. Il est d’ailleurs à noter que le «paradoxe du vote» n'existe que dans l'esprit de ceux qui sont attachés de façon inconditionnelle au paradigme utilitariste. Car, pour les autres, le vote n’a rien de mystérieux.

L'explication la plus simple du vote est en effet que l'électeur moyen perçoit les institutions démocratiques comme légitimes et qu'il les applique par souci de cohérence entre ses principes et son comportement: sous l'empire en d'autres termes de la «rationalité axiologique». Et lorsqu'il s'abstient, ce n'est pas parce que son vote est sans effet, mais plutôt, soit parce qu'il n'apprécie pas les candidats en présence ou les programmes qui lui sont soumis, soit parce qu'il se sent incapable de trancher entre eux, soit parce qu'il veut protester contre la consultation électorale, soit encore parce qu'il est pris de dégoût pour la politique ou pour d'autres raisons. Mais il est douteux qu'il faille voir dans les abstentionnistes des individus «rationnels» et dans les votants des individus «irrationnels ».

Pour ma part, je n’ai rencontré dans ma vie qu’un seul abstentionniste «rationnel» au sens de la théorie utilitariste du vote: un collègue original, qui est à ce point convaincu de la validité des théories utilitaristes, qu’il a décidé de s'y conformer rigoureusement. Il a des convictions politiques fortes, mais il ne vote jamais.

\section{RATIONALITÉ COGNITIVE ET RATIONALITÉ AXIOLOGIQUE}

Il résulte de cette discussion que, dans le cas du vote comme dans celui de la corruption, on n'est nullement condamné à choisir entre une explication relevant de la rationalité instrumentale («logique») et une explication de caractère irrationnel («non-logique »). Un jugement collectif de type «ceci est bon, légitime, etc.» peut en effet être fondé sur des raisons non conséquentialistes et a fortiori non instrumentales, sans que ces raisons soient illusoires et relèvent de la «rationalisation» au sens de Sigmund Freud ou de la «justifica- 
tion » (au sens dubitatif du terme). Ces raisons non conséquentialistes peuvent être la cause réelle de l'appréciation collective. Nul besoin ici d'évoquer de très conjecturaux «sentiments » ou de se contenter d'évoquer l'influence des effets de socialisation. Ces effets existent bien sûr, mais on ne peut les concevoir comme la seule source des jugements émis par le sujet. Je crois que 2 et 2 font 4 , non seulement parce qu'on me l'a appris, mais parce que 2 et 2 font effectivement 4. De même, ce n'est pas seulement parce qu'on me l'a appris que j'endosse l'idée que la corruption est mauvaise.

D'où proviendraient d'ailleurs le changement, le refus, la révolte, l'innovation dans un monde où l'individu se contenterait d'accepter les valeurs que la socialisation lui a transmises?

La faiblesse des théories irrationnelles du comportement utilisées dans les sciences sociales explique sans doute pour une part l'effort déployé, notamment par les économistes et par les sociologues se recommandant du modèle de l'utilité espérée (Rational Choice Theory), pour étendre la capacité explicative de la rationalité instrumentale. Cet effort a produit des résultats importants et parfois impressionnants s'agissant de maints sujets. Qu'on pense ici aux travaux de Mancur Olson (1978 [1965]), de Samuel Popkin (1979), de James Buchanan et Gordon Tullock (1965), de Richard McKenzie et Gordon Tullock (1975), de James Coleman (1990) ou de Timur Kuran (1995). Plusieurs chapitres de la sociologie - la sociologie du crime, la sociologie politique, la sociologie de l'opinion, la sociologie des mouvements sociaux, etc. - ont bénéficié de ces travaux.

Mais le nouvel utilitarisme ${ }^{5}$ n'est guère efficace s'agissant d'expliquer les phénomènes relevant notamment de l'ordre du normatif et de l'axiologique. L'utilitariste pourra toujours répliquer que celui qui se conforme aux principes qu'il estime fondés ne fait que chercher à maximiser l'estime de soi. Mais cette réponse ne saurait convaincre que les convaincus.

Bref, la distinction de Pareto continue de structurer les sciences sociales et humaines. Elle occupe un point stratégique dans la carte de ces disciplines. 
Le moment est peut-être venu de reconnaître qu'on ouvre des pistes prometteuses en prenant au sérieux le fait que la rationalité ne se réduit pas à la rationalité instrumentale. Lorsque les philosophes et les historiens des sciences parlent de «rationalité », ils veulent indiquer par là qu’on endosse une théorie lorsqu'on a des raisons de le faire. La rationalité qu’ils évoquent ainsi peut être qualifiée de «cognitive». C’est ce type de rationalité qui explique le rejet de la corruption ou le vote: les deux attitudes sont fondées sur une théorie plus ou moins clairement perçue par le sujet social, mais dont il ressent qu'il a des raisons d'y adhérer. On peut qualifier la rationalité «cognitive» d'«axiologique», dès lors qu'on l'applique au normatif.

Resterait à développer un point que j’ai eu l'occasion de soulever ailleurs, comme je l'indiquais en commençant. Le Traité de sociologie générale consacre ses plus longs développements à la théorie des «dérivations ». Celle-ci implique que les raisons prétendument fallacieuses que l'acteur social se donne, selon Pareto, pour «justifier» (au sens dubitatif») ses propres croyances à ses yeux et aux yeux des autres ne sont pas arbitraires. Au contraire, la conviction ne peut se former que si les raisons que les sujets se donnent sont articulées selon des règles strictes : celles que la théorie parétienne des «dérivations » s'efforce de mettre en évidence. Il y a des argumentations qui convainquent et d'autres non. Peut-on alors éviter de conclure que les premières convainquent parce qu'elles sont objectivement meilleures? Mais que reste-t-il alors de la conjecture parétienne selon laquelle toute argumentation échappant au cadre de pensée «logico-expérimental» (i.e. instrumentale conséquentialiste) serait par nature fallacieuse et généralement dépourvue d’influence causale réelle sur les croyances et les actions des hommes? On est bien en définitive obligé de conclure à l'existence d'une tension - pour ne pas parler de contradiction - entre la théorie des «résidus » ou des «sentiments » et la théorie des « dérivations ». 


\section{BIBLIOGRAPHIE}

ALBERT H., 1997, «The Conflict of Science and Religion: Religious Metaphysics and the Scientific View of Alternatives », Journal of Theoretical and Institutional Economics, I53-I, p. 217-232.

BOUDON R., 1984, «Le phénomène idéologique: en marge d'une lecture de Pareto», L'Année sociologique, 34, p.87-126.

-, 1995, Le Juste et le Vrai. Études sur l'objectivité des valeurs et de la connaissance, Paris, Fayard.

-, 1997, «Le "paradoxe du vote" et la théorie de la rationalité», Revue française de sociologie, 38-2, p. 217-227.

-, 2011, Croire et Savoir, Paris, PUF.

-, 2014, Le Rouet de Montaigne, Paris, Hermann.

BUCHANAN J., TULLOCK G.,1965, The Calculus of Consent, Ann Arbor, University of Michigan Press.

COLEMAN J. S.,1990, Foundations of Social Theory, Cambridge/London, The Belknap Press of Harvard University Press.

DEMEULENAERE P., 1996, Homo Fronomicus, Paris, PUF.

EVANS-PRITCHARD E. E., 1972 (1937), Sorcellerie, oracles et magie chez les Azandé, Paris, Gallimard.

GEERTZ C., 1984, «Anti Anti-Relativism», American Anthropologist, 86-2, p. 263-278.

HORTON R., 1982, «Tradition and Modernity Revisited», in Rationality and Relativism, M. Hollis, S. Luke (eds), Oxford, Blackwell, p. 201-260.

KURAN T., 1995, Private Truths, Public Lies. The Social Consequences of Preference Falsification, Cambridge Mass., Harvard University Press.

MCKENZIE R. B., TULLOCK G., 1975, The New World of Economics: Explorations into the Human Experience, Homewood, III., Irwin.

OLSON M., 1978 (1965), La Logique de l'action collective, Paris, PUF.

PARETO V.,1968 (1916), Traité de sociologie générale, Euvres complètes, t.XII, Genève, Droz. 
POPKIN S., 1979, The Rational Peasant, Berkeley, University of California Press.

SHWEDER R. A., 1977, «Likeliness and Likelihood in Everyday Thought: Magical Thinking in Judgments about Personality », Current Anthropology, 18-4, p. 637-665.

SPERBER D., 1997, «Individualisme méthodologique et cognitivisme», in Cognition et sciences sociales, R. Boudon, A. Bouvier, F. Chazel (éds), Paris, PUF, p. 123-136.

SPIRO M., 1995 (1987), Culture et Nature humaine, Paris, PUF.

STEINER Ph., 1998, Sociologie de la connaissance économique: essais sur les rationalisations de la connaissance économique, 1750-1850, Paris, PUF.

SUKALE M., 2002, Max Weber: Leidenschaft und Disziplin - Leben, Werk, Zeitgenossen, Tübingen, JCB Mohr (P. Siebeck).

VEYNE P., 1983, Les Grecs ont-ils cru à leurs mythes?, Paris, Seuil.

WEBER M., 1920-1921, Gesammelte Aufsätze zur Religionssoziologie, 3 vols, Tübingen, JCB Mohr (P. Siebeck).

-, 197I (1922), Économie et Société, Paris, Plon.

WITTGENSTEIN L., 1975, «Bemerkungen über Frazer's The Golden Bough», in Sprachanalyse und Soziologie, R. Wiggershaus (éd.), Frankfurt, Suhrkamp, p. 37-58. 